\title{
Suspension of Glycosaminoglycans in Oleogel Environment and Application Perspective in Gcmaf Immunotherapy
}

\author{
Comenius University, Faculty of Pharmacy, \\ Department of Galenic Pharmacy, Odbojárov 10 \\ SK-832 32 Bratislava
}

Received 14 June, 2021, accepted 23 June, 2021

Abstract The aim of this work was to find out the optimal concentration of gelling agents or their optimal combination in the olive oil environment to stabilise the suspension of chondroitin sulphate. Purified olive oil was structured with rice bran wax or a combination of gelators - soy lecithin and sorbitan tristearate. Rice bran wax creates a viscous fluid without a gel structure. The combination of soy lecithin and sorbitan tristearate forms a gel structure from $5 \mathrm{wt} . \%$ of the concentration. The optimal ratio of soy lecithin and sorbitan tristearate was found to be $3: 2 \mathrm{wt} . \%$. Oleogel was immobile at temperatures below $8^{\circ} \mathrm{C}$; on increasing the temperature, it changed to a viscous liquid. The oleogel environment opens new possibilities for creating oral route immunotherapy approaches such as glycoprotein-derived macrophage activating factor-derived immunotherapy.

Keywords oleogel-edible oleogelators-lecithin-chondroitin sulphate suspension

\section{INTRODUCTION}

The gel structure of dispersions is usually subjected to a hydrophilic environment. The same structure can be achieved in a lipophilic, non-polar environment. Research on organogelling hydrophobic solvents has increased sharply in recent years. The reason is the use of several new technological and biogalenic aspects.

The organogels are solid-like systems characterised by an organic fluid which is physically immobilised by a network of dispersed, aggregated gelator molecules. They are also named oleogels when the solvent is an edible oil (Co and Marangoni, 2012).

Organogels bring the possibility of improving the bioavailability of lipophilic drugs, improving the physical stability of oil suspensions, forming a stable environment against bacterial growth and avoiding the need to use antibacterial substances. In some cases, the organogel itself may be part of the pharmacological effect of the drug. An example of a triacylglycerol oleophilic environment is olive oil with a high (up to $75 \%$ ) oleic acid content (Preedy and Watson, 2020)

Organogelators represent a wide group of substances; but in pharmaceutical technology, their choice is considerably limited concerning the absence of potential toxic effects on the organism.

The criterion for selecting a suitable organogelator is the lowest concentration of a gel structure as possible. Suitable organogelling combinations can be achieved by a comprehensive gel-forming effect and a total reduction in the concentration of the substances used.

The organogelators as the structurant should meet the criteria of plasticity and stiffness at a defined temperature. In pharmaceutical applications, the gel structure should be maintained at a defined storage temperature, while the structure should disintegrate at the human body temperature. Pharmaceutically acceptable organogulators can be classified into two base groups such as polymer and monomeric organogelators. The polymeric organogelators include ethylcellulose. So far, only one polymer suitable for gelling of vegetable oils has been described. Ethylcellulose was found to generate gels in multiple hydrophobic solvents, including triacylglycerols (Co and Marangoni, 2012). Monomeric organogulators are richer and are represented as phospholipids, lecithins, fatty acid monoesters with glycerol, vegetable waxes (candelilic, carnaube wax and wax from rice 
brans), ceramides, phytosterols, fatty acid and sorbitol, fatty acid and protein hydroxyderivates, and proteins (Patel et al., 2015; Ghan et al., 2020; Rogers et al., 2014; Cerqueira et al., 2017).

Lecithins include widely available natural substances with gelating properties in oily environments. They do not create a gel structure themselves, but contribute to increasing the environmental viscosity. However, in combination with sorbitan fatty acid esters, they are a very effective complex organogulator with low minimal gelating concentration. At the same time, they are pharmacologically indifferent [Ruggero, 2005; Bhatnagar and Vyas, 1993].

\section{MATERIALS AND METHODS}

In the present work, purified purified olive oil was structured with rice bran wax or a combination of gelators soy lecithin (SL) and sorbitan tristearate (STS). As the solid phase of suspensions, chondroitin sulphate (CS) powder was used to study the kinetic stability.

The suspensions were prepared in two steps. The first step was preparation of the rice bran or SL and STS dispersions in purified olive oil by short-term heating to $80^{\circ} \mathrm{C}$ and subsequent dispersion by a high-speed mixing device. After cooling to room temperature, the solid phase, CS, was dispersed. The CS powder used had a particle size of up to $100 \mathrm{mcm}$.

\section{RESULTS AND DISCUSSION}

It was found that the rice bran wax creates viscous fluid without creating a gel structure. It did not reach the criteria for an oleogelling agent in a desirable concentration. The combination of SL and STS forms a gel structure from a total of
5 wt.\% of the concentration. The gel structure was significantly dependent on the temperature. The experiment detected by the optimal ratio of SL and STS gelector was 3:2 wt.\% dissolved in purified olive oil. The prepared oleogel was immobile at a temperature of $8^{\circ} \mathrm{C}$; on increasing the temperature gradually, it changed to semi-treated viscous liquid with a reduced viscosity. Above $25^{\circ} \mathrm{C}$, mild sedimentation was observed with the emergence of lightweight sediment.

The conversion of gel structure into semi-flowing viscous liquid on changing temperature offers a number of advantages to the pharmaceutical formulation in practice. The suspension is located in an immobile gel structure during the long-term storage, which prevents sedimentation of the solid phase. At the time of use, the formulation is allowed to warm to normal ambient temperature, which allows rapid changes in the gel structure, converting it into a viscous liquid. The liquid state allows short shaking and easy emptying of the content of the container at the time of use.

The oleogel environment is advantageous in the design of the dosage form in terms of improving the absorption of lipophilic substances as well as product stability. It opens new possibilities for creating new oral route immunotherapy approaches such as glycoprotein-derived macrophageactivating factor (GcMAF)-derived immunotherapy.

Oleogel provides a microbiologically stable environment and protects the suspended substances from moisture and oxygen. It can be widely used for dermal administration of medicinal substances in a solution or in an emulsion form.

\section{LIST OF ABBREVIATIONS}

SL - soy lecithin, STS - sorbitan tristearate, GcMAF glycoprotein-derived macrophage-activating factor

[7] Rogers MA, et al. Crystalline stability of self-assembled fibrillar networks of 12-hydroxystearic acid in edible oils. Int J Gastronomy Food Sci, 2014,2:22-31.

[8] Ruggero A et al. Biocompatible Lecithin Organogels: Structure and Phase Equilibria. Langmuir, 2005,21,140-148. 